\title{
Novel compound heterozygous mutations in CNGA1 in a Chinese family affected with autosomal recessive retinitis pigmentosa by targeted sequencing
}

\author{
Min Wang ${ }^{* \dagger}$, Dekang Gan ${ }^{\dagger}$, Xin Huang and Gezhi Xu
}

\begin{abstract}
Background: About 37 genes have been reported to be involved in autosomal recessive retinitis pigmentosa, a hereditary retinal disease. However, causative genes remain unclear in a lot of cases.

Methods: Two sibs of a Chinese family with ocular disease were diagnosed in Eye and ENT Hospital of Fudan University. Targeted sequencing performed on proband to screen pathogenic mutations. PCR combined Sanger sequencing then performed on eight family members including two affected and six unaffected individuals to determine whether mutations cosegregate with disease.

Results: Two affected members exhibited clinical features that fit the criteria of autosomal recessive retinitis pigmentosa. Two heterozygous mutations (NM000087, p.Y82X and p.L89fs) in CNGA1 were revealed on proband. Affected members were compound heterozygotes for the two mutations whereas unaffected members either had no mutation or were heterozygote carriers for only one of the two mutations. That is, these mutations cosegregate with autosomal recessive retinitis pigmentosa.

Conclusions: Compound heterozygous mutations (NM000087, p.Y82X and p.L89fs) in exon 6 of CNGA1are pathogenic mutations in this Chinese family. Of which, p.Y82X is firstly reported in patient with autosomal recessive retinitis pigmentosa.
\end{abstract}

Keywords: CNGA1, Autosomal recessive retinitis pigmentosa, Targeted exome sequencing

\section{Background}

Retinitis pigmentosa (RP, OMIM 268000) refers to a set of hereditary retinal diseases that feature progressive degeneration of the rod and cone photoreceptors. The classic symptom of RP includes early night blindness, progressive increase of the vision field constriction, pigment accumulation in the outer retina and gradually reduced visual acuity. As RP advances, patients will be eventually complete blindness. Nutritional supplementary of vitamin A and docosahexaenoic acid (DHA) in early, could slow progression of disease in many patients [1-4].

\footnotetext{
* Correspondence: ykmarkwang@163.com

${ }^{\dagger}$ Equal contributors

Department of Ophthalmology, Eye and ENT Hospital of Fudan University, Shanghai, China
}

(c) 2016 The Author(s). Open Access This article is distributed under the terms of the Creative Commons Attribution 4.0 International License (http://creativecommons.org/licenses/by/4.0/), which permits unrestricted use, distribution, and reproduction in any medium, provided you give appropriate credit to the original author(s) and the source, provide a link to the Creative Commons license, and indicate if changes were made. The Creative Commons Public Domain Dedication waiver (http://creativecommons.org/publicdomain/zero/1.0/) applies to the data made available in this article, unless otherwise stated.
RP can be inherited in three patterns-autosomal dominant, autosomal recessive and X-linked which account for $30 \sim 40 \%, 50 \sim 60$ and $5 \sim 15 \%$ of the RP patients, respectively [5-7]. Digenic inheritance and maternal (mitochondrial) inheritance have also been reported in few cases $[8,9]$. By now, 37 genes have been reported to responsible for autosomal recessive retinitis pigmentosa (ARRP) (http://sph.uth.edu/retnet/). Each gene accounts for $0.6 \sim 20 \%$ of ARRP patients. These genes are mainly functioned in following pathways: phototransduction cascade, vitamin A metabolism, structural and cytoskeletal and signaling, cell-cell interaction and synaptic interaction [10]. Although so many genes are identified, causative genes remain unclear in a lot of cases.

Given the harmful of RP, it's urgent to diagnose as soon as possible. However, RP is highly complicated. It's 
difficult to diagnose only rely on clinical features for some cases. Molecular diagnosis is an effective supplementary diagnosis method. The traditional way, PCR combined Sanger sequencing, are time-consuming and costly if there are a lot of candidate genes need to be examined. With the advent of next generation sequencing (NGS) technology, it's fast to determine the genotype of a large set of genes in parallel. It's especially useful as the price decrease with the progression of this technology. Target sequencing is a kind of NGS technology which can rapidly capture the entire protein-coding sequence. $\mathrm{Nu}$ merous causative mutations were accurately and rapidly revealed by this method in recent years [11-14].

In this study, a proband of a Chinese family was subjected to target sequencing and 11 variants are identified as candidate pathogenic mutations. Then, pedigree analysis showed that only two compound heterozygous mutations (NM000087, p.Y82X and p.L89fs) in exon 6 of CNGA1 co-segregate with disease. Of which, p.L89fs was identified in ARRP Japanese patients [15]. P.Y82X is a novel mutation. We conclude that these mutations are responsible for ARRP in this family.

\section{Methods}

\section{Samples and clinical examination of the RP patients}

A Chinese family was recruited from Eye and ENT Hospital of Fudan University. The pedigree of this family exhibits ocular disease with a recessive inheritance pattern (Fig. 1). A total of eight members including two affected and six unaffected individuals from this family participated in this study. Before inclusion, complete ophthalmological examinations were carried out on the eight members. These included visual acuity testing (Snellen chart), computerized visual field measurement (Humphrey Visual Field Analyzer, Carl Zeiss Inc., CA, USA), tests of dark adaptation (ECLIPSE Dark Adaptometry), color vision (Ishihara color plate), spectral domain optical coherence tomography (SPECTRALIS ${ }^{\bullet}$ HRA + OCT, Heidelberg
Engineering Inc., Heidelberg, Germany) and fundus autofluoresence (SPECTRALIS ${ }^{\circ}$ HRA + OCT, Heidelberg Engineering Inc., Heidelberg, Germany). Besides, full field electroretinogram (LKC Utas E3000 LKC Technologies Inc., USA) was performed on two affected individuals (II1 and II3, Fig. 1). Medical history of the two affected individuals was also obtained regarding the following aspects: subjective degree of vision loss, age at onset, evolution, medication and other relevant clinical manifestations. In addition, a total of 400 unrelated volunteers from China were served as control group. These volunteers show normal phenotype in visual acuity testing and visual field measurement and don't have other obvious serious diseases. Blood samples were collected from the eight subjects and stored in $4{ }^{\circ} \mathrm{C}$ until analysis.

\section{DNA isolation and high-throughput sequencing}

Genomic DNA was extracted from peripheral blood according to manufacturer's instructions (QIAGEN, Hilden, Germany). DNA sequencing libraries were then prepared as followed according to Illumina standard protocol: genomic DNA was fragmented; Illumina adapters were ligated to the fragments after 'A' ligating to their 3'ends; Fragments with sample size in 200 to 500base pair were selected and amplified by PCR (each sample is tagged with a unique index during this procedure). Fragments in the exonic regions of targeted genes were captured by a specific Hereditary Ophthalmological Disease GenePanel using biotinylatedoligo-probes (MyGenosticsGenCap Enrichment Technologies, MyGenostics, Baltimore, MD, USA). The Panel was designed to detect the coding region of 371 genes which cover almost all of genes that reported to relate to hereditary ophthalmological disease (Additional file 1). The capture experiment was conducted according to the manufacturer's protocol. Briefly, $1 \mu \mathrm{g}$ DNA library was mixed with Buffer BL and GenCap gene panel probe. The mixture was heated at $95{ }^{\circ} \mathrm{C}$ for $7 \mathrm{~min}$ and $65{ }^{\circ} \mathrm{C}$ for $2 \mathrm{~min}$. Adding $23 \mu \mathrm{l}$ of the $65{ }^{\circ} \mathrm{C}$ prewarmed

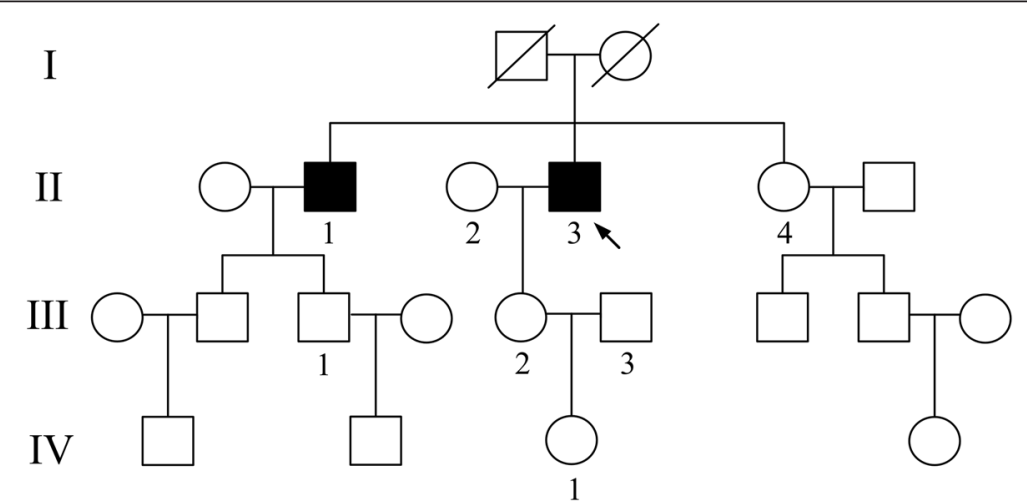

Fig. 1 Pedigree of the Chinese family. Circles and squares represent females and males, respectively. Two filled squares represent two patients with ARRP in this family. Proband is indicated by arrow. Subjects who participate in DNA analysis are labeled with numbers 
Buffer HY (MyGenostics) to mixture, then hybridizate at 65 ${ }^{\circ} \mathrm{C}$ for $22 \mathrm{~h}$. After adding $64 \mu \mathrm{l} 2 \mathrm{X}$ binding buffer and $80 \mu \mathrm{l}$ MyOnebeads(Life Technology), the hybrid mixture was transferred to the tube. The mixture was rotated and the beads were washed. The bound DNA was then eluted followed by amplification activated at $98{ }^{\circ} \mathrm{C}$ for $30 \mathrm{~s}(1$ cycle), $98{ }^{\circ} \mathrm{C}$ for $25 \mathrm{~s}, 65^{\circ} \mathrm{C}$ for $30 \mathrm{~s}, 72^{\circ} \mathrm{C}$ for $30 \mathrm{~s}(15 \mathrm{cy}-$ cles), $72{ }^{\circ} \mathrm{C}$ for $5 \mathrm{~min}$ ( 1 cycle). The PCR products were purified and then sequenced by IlluminaHiSeq ${ }^{\mathrm{Tm}} 2000$ sequencer, generating $2 \times 100$ bp reads. Base was called using the Off-Line Base Caller v1.9.

\section{Bioinformatics analysis}

Raw reads were firstly filtered out low quality reads and adaptor sequences using Trimmomatic [16]. The PCR duplicates were removed using the Picard software (http://broadinstitute.github.io/picard/). Then, clean reads were align to the human reference genome (hg19) using BWA [17]. Sequence variations including single nucleotide polymorphisms (SNPs) and insertions or deletions (InDels) were identified using the GATK program (https://www.broadinstitute.org/gatk/). A variant locus with at least five reads support and minor allele frequency $>0.3$ was kept in variant analysis. The identified SNPs and InDels were annotated using the ANNOVAR (http://annovar.openbioinformatics.org/en/latest/). IGV was used to view the short read alignment and validate the candidate SNPs and InDels (http://www.broadinstitute.org/igv/). The variants are further filtered to identify pathogenic mutations. A variant is determined as pathogenic mutation mainly based on the following three principals: 1) It predicted to alter the sequence of encoded protein, 2) it occurs in different frequency between case group and normal group, 3) it co-segregate with disease in family carrying it. Specifically, novel variants with synonymous effect or minor allele frequency (MAF) >0.01 in 1000 genome variants database or ESP6500 database are discarded. And for known variants, functional effect is not taken into consideration since variant with synonymous effect is yet reported. Only those variants with MAF $>0.05$ in 1000 genome variants database or ESP6500 database are discarded.

\section{Mutation analysis of CNGA1}

Two mutations in exon 6 of CNGA1 (NM 000087, c.265delC and c.246C > A) were determined by PCR combined with Sanger DNA sequencing in 8 family members. In brief, genomic DNA was extracted and amplified by PCR on standard condition. The primer sequences and precise PCR conditions are available from the authors on request. Undirectional sequencing was carried out using the forward primers. And if this failed, reverse primers were used to sequencing. Sanger sequencing was performed using the ABI PRISMBig Dye Terminator cycle sequencing ready reaction kit on a 3100 ABI DNA sequencer (Thermo-Fisher).

\section{Results}

\section{Clinical assessments}

Clinical characteristics of the two affected sibs in this family are listed in Table 1 . Both affected individuals suffer from night blindness in early childhood, progressive loss of visual fields and decreased visual acuity with age. All of the features fit the diagnostic criteria of RP.

Table 1 Clinical characteristics of two affected sibs in the family

\begin{tabular}{lll}
\hline Patient & II.3 & II.1 \\
\hline Onset (years) & & 9 \\
Night blindness & 5 & 24 \\
Visual field defect & 16 & 31 \\
$\begin{array}{l}\text { Decreased visual acuity } \\
\text { Ophthalmological }\end{array}$ & 20 & \\
examination & & \\
Visual acuity & RE CF/10 & RE 20/60 \\
& LE CF/20 & LE 20/80 \\
Slit lamp & Mild cataract & Normal \\
$\begin{array}{l}\text { Visual field } \\
\text { Fundus }\end{array}$ & RE/LE: not detectable & RE/LE: nasal and temporal defects \\
Optical coherence & atrophy, pale disc, arteriolar constriction & RE/LE: paravascular bone spicule pigmentation, RPE \\
tomography & RE/LE: extensive ellipsoid band loss, RPE thinning & atrophy, pale disc, arteriolar constriction \\
Fundus autofluorescence & RE/LE: extensive hypo-fluorescence in the posterior pole & RE/LE: peripheral ellipsoid band loss, peripheral RPE \\
Electroretinogram & RE/LE: extinguished & thinning \\
\hline
\end{tabular}

$R E$ right eye, $L E$ left eye, $C F$ counting finger, RPE retinal pigment epithelium 
Proband eventually completely lost of his sight when he was $\sim 50$. Furthermore, fundus examination of proband shows the typical RP appearance including paravascular bone spicule pigmentation, retinal pigmented epithelium atrophy, pale disc, and arteriolar constriction (Fig. 2). Optical coherence tomography (OCT) showed both eyes of proband had a similar change as shown in Fig. 2. That is, severe ellipsoid band loss in outer retina, obvious loss of retinal nerve fiber, thin retinal pigment epithelium (RPE) and choroid thickness. Meanwhile, both eyes of proband had completely loss of retinal nerve fiber layer (RNFL) without enlargement of cup/disc ratio. The six unaffected individuals showed normal phenotype in all the ophthalmological examinations except that III1 and III2 were suffering from myopia.

\section{Pathogenic mutation detection}

Targeted sequencing is performed on Proband (II3) using a specific Hereditary Ophthalmological Disease GenePanel. This gene panel includes almost all genes that have been reported to relate to ophthalmological diseases. In total, 5,160, $906 * 2$ pair-end reads are generated. $99 \%$ of targeted regions are sequenced, reaching an average depth of $\sim 297$ fold which allowed to accurately identification of a SNP. Specifically, the average read depth of ARRP genes is $\sim 311$ fold, rang from 56 to 948 . A total of 502 variants, including 494 SNVs and eight Indels are identified in target regions. According to the functional effects, the variants are categorized as synonymous, non-synonymous, splice-site, stop-gain, frameshift, non-frameshift and unknown group.

Eleven variants are identified as candidate pathogenic mutations after filtering step as described in methods. Of which, three variants (ATXN1:NM_000332:p.E357K,
TCF4:NM_001243226:c.1793-5G > A and KRT3:NM_057 088:c.1189-5T >C) are known pathogenic variants of dominant retinitis pigmentosa. Two variants (CNGA1: NM_000087:Y82X and CNGA1: NM_000087:L89fs) are involved in ARRP. And the rest variants (USH2A:NM_206933:c.11549-5T > -, COL4A3:NM_000091:p.I156 7S，TLR4:NM_003266:p.E434K，INPP5E:NM_019892:p.Q 633E, HMCN1:NM_031935:p.V170I and KRT6B:NM_005 555:p.V454I) are involved in other type of ophthalmological diseases. To determine which variants are the pathogenic mutations, co-segregation analysis is performed on 8 members of this family (II1, II2, II3, II4, III1, III2, III3 and IV1, Fig. 1). Result shows that only the two compound heterozygous mutations in CNGA1co-segregate with this disease (Additional files 2 and 3). Both of the two affected individuals (II1 and II3) carry two heterozygous variants. Two unaffected individuals (III1 and III2) only carry one of the two heterozygous variants. And the other four unaffected individuals (II2, II4, III3 and IV1) are in wild type for the two loci (Fig. 3). Furthermore, the frequency of the two mutations is 0.0074 and 0 in the control group consists of 400 unrelated healthy individuals, respectively (Table 2). Based on mutation analysis and the clinical assessment, we conclude that the compound heterozygous mutations are the pathogenic variants of ARRP in this family.

CNGA1 was reported as a causative gene of ARRP [18]. The first mutation (CNGA1: NM_000087:L89fs) leads a frame shift from residue 89 of the protein, which was reported in ARRP Japanese patient. And the second one (CNGA1: NM_000087:Y82X), a novel mutation, leads to generate a premature termination codon at residue 82 . Both of the two mutations occur early in the reading frame that clearly would change the function of the protein.

a
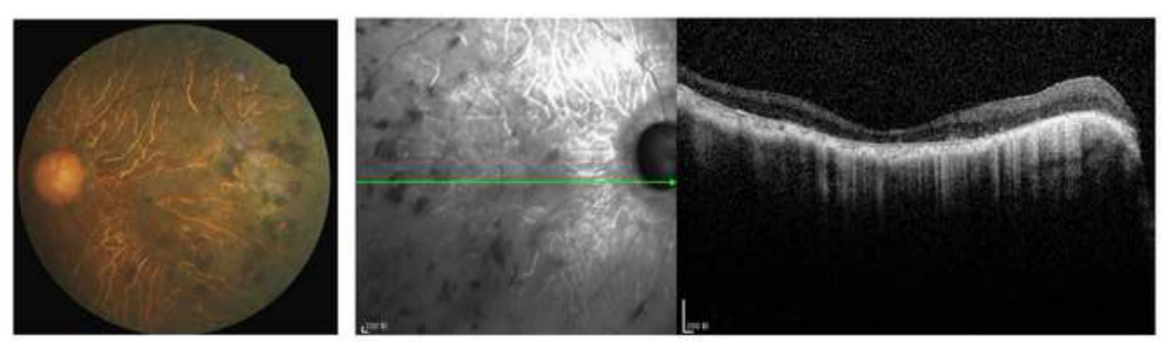

b
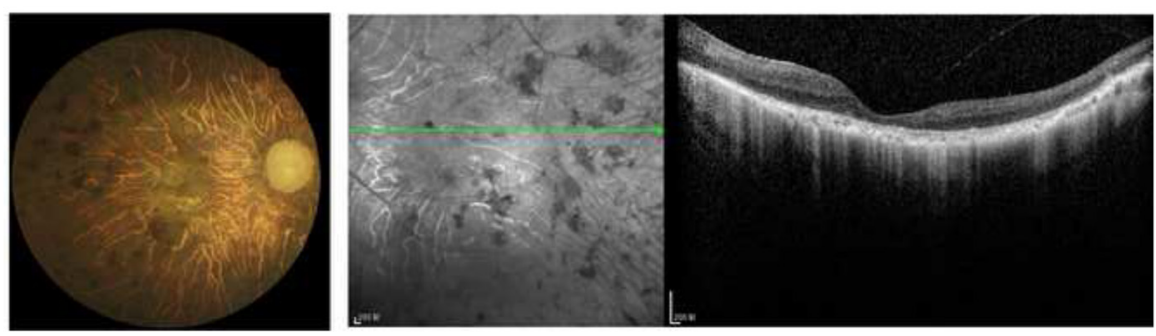

Fig. 2 Fundus and OCT images. Fundus and OCT photographs from proband show typical changes of RP. a: right eye b: left eye 
CNGAl c.265delC

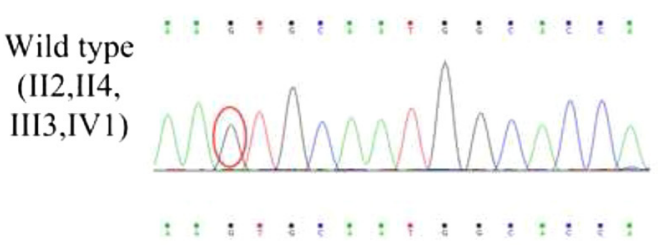

Carrier

(III2)
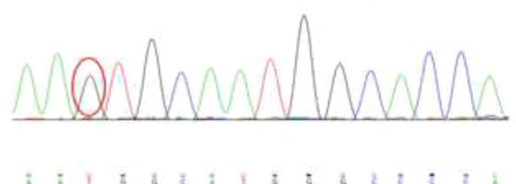

Carrier

(III1)

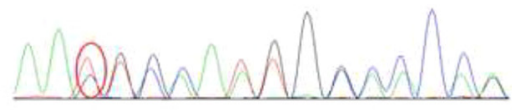

Affected

(II1,II3)
CNGAl c. $246 \mathrm{C}>\mathrm{A}$
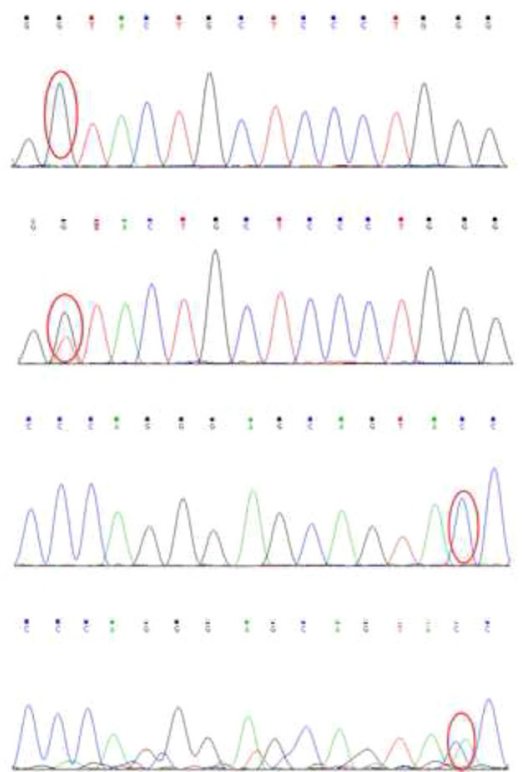

Fig. 3 CNGA1 sequence variation analysis. CNGA1 sequence variation pattern are clarified into three types: wild type, no mutation in the two loci; carrier, only one of the two loci in mutant status; affected: two loci were in mutant status. Individuals belong to each type are presented in parenthesis

\section{Discussion}

CNGA1 encodes a protein-a subunit of the rod cGMPgated channel, which involved in the phototransduction pathway [19]. Functional protein of CNGA1 consists of six putative transmembrane domains and a pore region. Several mutations in CNGA1 have been revealed to be pathogenic mutations for ARRP by now [15, 18, 20-23]. In which, mutations that lead to deletion of transmembrane domains were reported to be totally nonfunctional. Protein which contains mutations that leads to deletion of C-terminal 32 residues can't locate to cell membrane although the normal channel activity still maintained. The two mutations (c.265delC and c.246C > A) that we identified in this study occur early in the reading frame which lead to lost most of coding region including all transmembrane domains. It is speculated that no functional CNGA1 protein was generated in affected individuals of this family.

So far, several studies reported RP patients with CNGA1 mutations. All patients including the two sibs we reported here generally showed typical characteristics of RP $[15,20,24,25]$. For example, they noticed night blindness in early childhood, gradually lost visual field and decreased in visual acuity. Most of them had a severely constrained peripheral visual field. Only one patient showed ring scotoma with a preserved peripheral visual field [15]. In our study, II.1 had a nasal and temporal visual field and II.3 lost all of visual field. Noticeably, II.3 was observed to suffer mild cataract under slitlamp testing. This was not reported in other patients with CNGA1 mutation. The fundus images of all the patients showed pale optic discs, attenuation of retinal vessels, bone spicule pigmentation and retinal pigment epithelium atrophy. Some of the patients had normal macular region and some of them were identified with retinal degeneration in macular regions. In our study, II.1 showed an abnormal high density ring in macula in the image of fundus autofluorescence which was also observed in a patient from China [24]. Both of the two affected sibs showed extinguished ERG signal which was common in other studies. When scanned by spectral domain optical coherence tomography (SD-OCT), the proband showed unusual retinal changes. That is, both eyes had completely loss of retinal nerve fiber layer (RNFL) without enlargement of cup/disc ratio. This feature was unusual because many RP patients had

Table 2 CGNA1 mutations detected in proband

\begin{tabular}{|c|c|c|c|c|c|c|c|}
\hline Gene symbol & Position & Transcript ID & Exon NO. & DNA change & Protein change & Hom/Het & Frequency \\
\hline CNGA1 & chr4 47951884-47951885 & NM_000087 & exon 6 & c.265delC & p.L89fs & het & 0.0074 \\
\hline CNGA1 & chr4 47951903 & NM_000087 & exon 6 & c. $246 C>A$ & p.Y82X & het & 0 \\
\hline
\end{tabular}

Hom homozygous, Het heterozygous, Frequency: mutation frequency in control group of 400 healthy individuals 
thicker RNFL even in the late stage of the disease [26]. We assume that this type of genetic mutation may lead to severer optic nerve atrophy than other genotype.

About $2.5 \%$ patients with ARRP are caused by mutations in CNGA1 in western population [25]. Only few studies reported the genetic causes of ARRP in Chinese population [24, 27]. Here we reports mutations in CNGA1 responsible for ARRP in Chinese population, which expand our knowledge about APPR in Chinese population.

\section{Conclusion}

In this study, we descried a Chinese family in which two sibs suffer from severe ocular disease. Proband even completely losses his sight. Disease was transmmitted via autosomal recessive pattern in this family. Clinical measurements show that their symptom fit the criteria of ARRP. By screening 371 genes which have been reported to contribute to hereditary eye disease, two compound heterozygous mutations in the exon 6 of CNGA1 (c.265delC and c.246C > A) were revealed. Furthermore, pedigree analysis was shown that the heterozygous mutations co-segregate with disease. Together, the results demonstrate that the compound heterozygous mutations are the pathogenic mutations of ARRP in this family. Of the two mutations, c. $246 \mathrm{C}>\mathrm{A}$ is a novel mutation.

\section{Additional files}

Additional file 1: Gene symbol list that include in the Hereditary Ophthalmological Disease GenePanel. (XLSX 12 kb)

Additional file 2: The status of nine mutations in eight family members which are ruled out from pathogenic mutations. (XLSX 8 kb)

Additional file 3: Sequencing image of nine mutations in eight family members which are ruled out from pathogenic mutations. (PDF $159 \mathrm{~kb}$ )

\footnotetext{
Abbreviations

ARRP, Autosomal recessive retinitis pigmentosa; NGS, Next generation sequencing
}

\section{Acknowledgements}

None.

\section{Funding}

This research project was supported by Natural Science Foundation of Shanghai (Grant code 13ZR1406100)

\section{Availability of data and materials}

Data can be shared upon request. Mail: ykmarkwang@163.com.

\section{Authors' contributions}

MW and DG conceived this study, determined the pathogenic mutations and wrote this manuscript. XH and GX gave assistance in sample collecting and performed PCR assays. All authors read and approved the final manuscript.

\section{Competing interests}

The authors declare that they have no competing interests.

\section{Consent for publication}

Informed consent to publish was obtained from every subject before inclusion.

\section{Ethics approval and consent to participate}

This study followed the tenets of the Declaration of Helsinki and was approved by the ethics committee of Eye and ENT Hospital of Fudan University. Informed consent was obtained from every subject before inclusion.

Received: 10 November 2015 Accepted: 16 June 2016

Published online: 08 July 2016

\section{References}

1. Berson EL, Rosner B, Sandberg MA, Hayes KC, Nicholson BW, Weigel-DiFrano C, Willett W. Vitamin A supplementation for retinitis pigmentosa. Arch Ophthalmol. 1993;111:1456-9.

2. Sacchetti M, Mantelli F, Merlo D, Lambiase A. Systematic Review of Randomized Clinical Trials on Safety and Efficacy of Pharmacological and Nonpharmacological Treatments for Retinitis Pigmentosa. J Ophthalmol. 2015:2015:737053.

3. DR H m, Locke KG, Wheaton DH, Fish GE, Spencer R, Birch DG. A randomized, placebo-controlled clinical trial of docosahexaenoic acid supplementation for X-linked retinitis pigmentosa. Am J Ophthalmol. 2004:137:704-18.

4. Berson EL, Rosner B, Sandberg MA, Weigel-DiFranco C, Moser A, Brockhurst $R J$, et al. Further evaluation of docosahexaenoic acid in patients with retinitis pigmentosa receiving vitamin A treatment: subgroup analyses. Arch Ophthalmol. 2004:122:1306-14.

5. Bunker $\mathrm{CH}$, Berson EL, Bromley WC, Hayes RP, Roderick TH. Prevalence of retinitis pigmentosa in Maine. Am J Ophthalmol. 1984;97:357-65.

6. Grondahl J. Estimation of prognosis and prevalence of retinitis pigmentosa and Usher syndrome in Norway. Clin Genet. 1987;31:255-64.

7. Pawlyk BS, Bulgakov OV, Sun X, Adamian M, Shu X, Smith AJ, Berson EL, Ali RR, Khani S, Wright AF, Sandberg MA, Li T. Photoreceptor rescue by an abbreviated human RPGR gene in a murine model of $X$-linked retinitis pigmentosa. Gene Ther. 2016;23(2):196-204.

8. Dryja TP, Hahn LB, Kajiwara K, Berson EL. Dominant and digenic mutations in the peripherin/RDS and ROM1 genes in retinitis pigmentosa. Invest Ophthalmol Vis Sci. 1997:38:1972-82.

9. Kajiwara K, Berson EL, Dryja TP. Digenic retinitis pigmentosa due tomutations at the unlinked peripherin/RDS and ROM1 loci. Science. 1994;264:1604-8.

10. Hartong DT, Berson EL, Dryja TP. Retinitis pigmentosa. Lancet. 2006;368: 1795-809.

11. Simpson DA, Clark GR, Alexander S, Silvestri G, Willoughby CE. Molecular diagnosis for heterogeneous genetic diseases with targeted high-throughput DNA sequencing applied to retinitis pigmentosa. J Med Genet. 2011:48:145-51.

12. Neveling $K$, Collin RW, Gilissen $C$, van Huet RA, Visser L, Kwint MP, et al. Next-generation genetic testing for retinitis pigmentosa. Hum Mutat. 2012; 33(6):963-72.

13. Tucker BA, Scheetz TE, Mullins RF, DeLuca AP, Hoffmann JM, Johnston RM, et al. Exome sequencing and analysis of induced pluripotent stem cells identify the cilia-related gene male germ cell-associated kinase (MAK) as a cause of retinitis pigmentosa. PNAS. 2011;108(34):569-76.

14. Sun $Y$, Zhang Z, Cheng J, Lu Y, Yang CL, Luo YY, et al. A novel mutation of EYA4 in a large Chinese family with autosomal dominant middle-frequency sensorineural hearing loss by targeted exome sequencing. J Hum Genet. 2015;60(6):299-304.

15. Katagiri S, Akahori M, Sergeev Y, Yoshitake K, Ikeo K, Furuno M, et al. Whole exome analysis identifies frequent CNGA1 mutations in Japanese population with autosomal recessive retinitis pigmentosa. PLoS ONE. 2014; 9(9):e108721.

16. Bolger AM, Lohse $M$, Usadel B. Trimmomatic: A flexible trimmer for Illumina Sequence Data. Bioinformatics. 2014:30(15):2114-20.

17. Li H, Durbin R. Fast and accurate long-read alignment with Burrows-Wheeler Transform. Bioinformatics. 2010;26(5):589-95.

18. Dryja TP, Finn JT, Peng YW, McGee TL, Berson EL, Yau KW. Mutations in the gene encoding the a subunit of the rod CGMP-gated channel in autosomal recessive retiiitis pigmentosa. Proc Natl Acad Sci. 1995;92(22):10177-81. 
19. Yau KW. Phototransduction mechanism in retinal rods and cones. The Friedenwald Lecture. Invest Ophthalmol Vis Sci. 1994;35(1):9-32

20. Paloma E, Martínez-Mir A, García-Sandoval B, Ayuso C, Vilageliu L, Gonzàlez-Duarte R, et al. Novel homozygous mutation in the alpha subunit of the rod CGMP gated channel (CNGA1) in two Spanish sibs affected with autosomal recessive retinitis pigmentosa. J Med Genet. 2002;39:e66.

21. Jin ZB, Mandai M, Yokota T, Higuchi K, Ohmori K, Ohtsuki F, et al. Identifying pathogenic genetic background of simplex or multiplex retinitis pigmentosa patients: a large scale mutation screening study. J Med Genet. 2008;45(7):465-72.

22. Eisenberger $T$, Neuhaus $C$, Khan $A O$, Decker $C$, Preising MN, Friedburg $C$, et al. Increasing the yield in targeted next-generation sequencing by implicating CNV analysis, non-coding exons and the overall variant load: the example of retinal dystrophies. PLoS ONE. 2013;8(11):e78496.

23. Wang F, Wang H, Tuan HF, Nguyen DH, Sun V, Keser V, et al. Next generation sequencing-based molecular diagnosis of retinitis pigmentosa: identification of a novel genotype-phenotype correlation and clinical refinements. Hum Genet. 2014;133(3):331-45.

24. Jin X, Qu LH, Hou BK, Xu HW, Meng XH, Pang CP, Yin ZQ. Novel compound heterozygous mutation in the CNGA1 gene underlie autosomal recessive retinitis pigmentosa in a Chinese family. Biosci Rep. 2016;22:36(1). doi:10. 1042/BSR20150131.

25. Zhang Q, Zulfiqar F, Riazuddin SA, Xiao X, Ahmad Z, Riazuddin S, et al. Autosomal recessive retinitis pigmentosa in a Pakistani family mapped to CNGA1 with identification of a novel mutation. Mol Vis. 2004;10:884-9.

26. Xue K, Wang M, Chen J, Huang X, Xu G. Retinal nerve fiber layer analysis with scanning laser polarimetry and RTVue-OCT in patients of retinitis pigmentosa. Ophthalmologica. 2013;229(1):38-42.

27. Xu Y, Guan L, Shen T, Zhang J, Xiao X, Jiang H, Li S, Yang J, Jia X, Yin Y, Guo $X$, Wang J, Zhang Q. Mutations of 60 known causative genes in 157 families with retinitis pigmentosa based on exome sequencing. Hum Genet. 2014; 133(10):1255-71.

\section{Submit your next manuscript to BioMed Central and we will help you at every step:}

- We accept pre-submission inquiries

- Our selector tool helps you to find the most relevant journal

- We provide round the clock customer support

- Convenient online submission

- Thorough peer review

- Inclusion in PubMed and all major indexing services

- Maximum visibility for your research

Submit your manuscript at www.biomedcentral.com/submit

C Biomed Central 